\title{
The Excitability of Human Corticospinal Neurons is Depressed by Thiopental
}

\author{
K. Kong, C. Ukachoke, G. McGuire, D. Wong and P. Ashby
}

\begin{abstract}
Background: We tested the effect of thiopental on the excitability of the corticospinalmotoneuron axis in normal human subjects. Methods: Magnetic stimulation was used to excite the neurons in the motor cortex which give rise to the fast conducting corticospinal pathway. The characteristics of the composite excitatory post-synaptic potentials (EPSPs) produced in individual spinal motoneurons by cortical stimulation were derived from changes in the firing probability of voluntarily activated motor units of the first dorsal interosseous muscle. Results: In 5 normal subjects, we found that thiopental, in incremental doses sufficient to sustain drowsiness (total dose 75 to $175 \mathrm{mg}$ ), significantly reduced the amplitude of these composite EPSPs. Conclusions: Thiopental reduced the facilitation of motoneurons from the cortex most likely by depressing cortical neurons.
\end{abstract}

RÉSUMÉ: L'excitabilité des neurones corticospinaux humains est déprimée par le thiopental. Introduction: Nous avons évalué l'effet du thiopental sur l'excitabilité de l'axe corticospinal-motoneurone chez des sujets humains. Méthodes: Nous avons utilisé une stimulation magnétique pour exciter les neurones du cortex moteur qui donnent naissance à la voie corticospinale à conduction rapide. Les caractéristiques des potentiels post-synaptiques excitateurs composés (EPSPs), produits dans les motoneurones spinaux individuels par stimulation corticale, provenaient de changements dans la probabilité de décharge d'unités motrices activées volontairement au niveau du premier muscle dorsal interosseux. Résultats: Chez 5 sujets normaux, nous avons constaté que le thiopental, utilisé en doses croissantes suffisantes pour maintenir la somnolence (dose totale de 75 à $175 \mathrm{mg}$ ), réduisait significativement l'amplitude de ces EPSPs composés. Conclusions: Le thiopental réduit la facilitation présynaptique des motoneurones du cortex, probablement par dépression des neurones corticaux.

Can. J. Neurol. Sci. 1995; 22: 218-222

The principal action of the anesthetic agent thiopental is considered to be enhancement of gamma-aminobutyric acid (GABA) mediated inhibition.' It is not known which populations of neurons in the central nervous system (CNS) are affected. The present study was undertaken to determine whether intravenous thiopental could be shown to alter the excitability of the corticospinal-motoneuron axis in humans.

Magnetic stimulation applied over the human motor cortex excites corticospinal neurons and causes a short latency facilitation of contralateral spinal motoneurons. ${ }^{2}$ The corticospinal neurons are excited both directly, ${ }^{3}$ and indirectly (that is transsynaptically). ${ }^{4}$ The descending volleys in corticospinal axons facilitate spinal motoneurons. The short latency and brief duration of the facilitation is compatible with the presence of monosynaptic projections to motoneurons. ${ }^{2.5}$ If the projections of corticospinal neurons to spinal motoneurons are monosynaptic, then the magnitude of the composite excitatory postsynaptic potential (EPSP) produced in a single spinal motoneuron by a given magnetic stimulus should reflect moment to moment changes in the excitability of the pool of cortical neurons activated by the magnetic stimulus. In man the characteristics of composite EPSPs in single spinal motoneurons can be derived from the changes in the firing probability of single voluntarily activated motor units, as measured in post-stimulus time histograms (PSTH). ${ }^{6}$ Thus changes in the firing probability of single motor units in response to magnetic stimulation over the cortex can provide an estimate of the excitability of the corticospinal-motoneuron axis..$^{7.8} \mathrm{We}$ used this method to investigate the action of thiopental.

\section{Methods}

Subjects

Six healthy subjects who had no allergy to thiopental volunteered for the study after providing informed consent. The study received approval from the local ethics committee.

\section{Magnetic stimulation}

A brief, rapidly changing magnetic field was generated with a Cadwell MES-10 magnetic stimulator. The stimulating coil was placed flat over the scalp, centered over C3 (a point $20 \%$ of the distance from the vertex on a line drawn between the vertex and the tragus of the right ear). The stimulus intensity was adjusted

From the Division of Neurology and Playfair Neuroscience Unit (K.K.. C.U.. P.A.), and Department of Anaesthesia (G.M., D.W.), University of Toronto. Toronto

RECEIVED APRIL, 18, 1994. ACCEPTED IN FINAL. FORM MARCH 15, 1995

Reprint requests to: Dr. Peter Ashby. Division of Neurology, Playfair Neuroscience Unit \#13-319, Toronto Western Hospital, 399 Bathurst Street, Toronto, Ontario, Canada M5T 2S8 
so that it was well below the intensity which resulted in a visible contraction of the weakly contracted first dorsal interosseous muscle of the left hand, but sufficient to cause short latency facilitation of voluntarily activated first dorsal interosseous muscle on a trial run. Stimuli were delivered to the scalp at random intervals between 3 and 5 seconds, with 100 stimuli in each run, so each run took approximately 7 minutes. The stimulus site and intensity remained constant throughout the entire recording session.

\section{Motor unit recordings}

A disposable, concentric needle electrode was inserted into the first dorsal interosseous muscle of the left hand, and positioned close to a motor unit activated by gentle voluntary contraction. The action potentials of this single motor unit were extracted with a window discriminator and displayed on a storage oscilloscope using a delay line. The pulse from the window discriminator was led to a loudspeaker and a tachometer. The subject was provided with visual and auditory feedback of the unit's discharges and its instantaneous firing rate and instructed to keep the single unit discharging at a constant rate.

The same motor unit was used for the entire study. With each run of 100 magnetic stimuli, a PSTH of the unit's discharge times was generated by a laboratory computer. A peak of increased firing probability in the PSTH was recognized where the bin contents of one or more adjacent bins exceeded the mean plus 2 standard deviations of the 200 bin pre-stimulus portion of the record. The area of the peak (number of additional counts above the prestimulus mean) was recorded and expressed as extra counts per 1,000 stimuli. For further details of the method see Mao et al. ${ }^{9}$ and Palmer and Ashby. ${ }^{5}$

\section{Protocol}

\section{Control run}

The subject kept the unit discharging regularly while 100 magnetic stimuli were delivered. The instantaneous firing rate was observed on the tachometer.

\section{Thiopental run(s)}

Intravenous thiopental was administered by an anaesthetist. An initial dose of $50 \mathrm{mg}$ was followed by increments of $25 \mathrm{mg}$ until the subject felt slightly drowsy and nystagmus was observed on lateral gaze. Then a further 100 magnetic stimuli were delivered while the subject maintained the firing of the motor unit as close to the previous rate as possible. During this run, further "top-up" doses of thiopental (in increments of 25 $\mathrm{mg}$ ) were given so that the subject was maintained in a slightly drowsy state. The total dose given ranged from $75-175 \mathrm{mg}$ (1.5$2.5 \mathrm{mg} / \mathrm{kg}$ ). Two subjects continued to feel the effects of the thiopental more than 10 minutes after the last dose was given. This provided an opportunity for a further run of 100 magnetic stimuli, and the mean result of the two experimental runs was used in the statistical analysis.

\section{Recovery $\operatorname{run}(s)$}

When the subject reported that they felt normal again, and no nystagmus was observed, another recording during 100 magnetic stimuli was completed. One subject reported feeling even more alert after his run was completed, and another recovery run was performed. The mean of the two recovery runs was used.

\section{Statistical Analysis}

The means of the areas and durations of the PSTH peaks, the duration of the subsequent period of decreased firing probability and the motor unit firing rates (calculated from the number of discharges per unit time) were compared between the control and recovery runs (pooled) and the run with thiopental with the Student's $t$ test of differences. Probabilities $<0.05$ were taken as significant.

\section{Results}

Successful studies were completed in 5 subjects; 3 males, 2 females, between 29 and 53 years of age (mean 39 yrs). In each of these studies all observations were made on a single motor unit. In a sixth subject a single unit could not be recorded in isolation for the required time and the study was discarded. An example of the changes in firing probability in a single motor unit produced by magnetic stimulation over the motor cortex is shown in Figure 1. In first run (control), the stimulus resulted in a period of increased firing probability which is represented in the PSTH by a cluster of extra counts occurring at a latency of $26 \mathrm{~ms}$. The area of the peak was 248 extra counts. During the administration of intravenous thiopental (a total dose $2.5 \mathrm{mg} / \mathrm{kg}$ was used), the peak area was 94 extra counts. During the recovery run, the peak area was 172 extra counts.

In the group of 5 subjects, intravenous thiopental resulted in a decrease in the area of the PSTH peaks produced by magnetic stimulation (mean peak areas, standard errors in brackets: control 337 (63), thiopental $168(28)$, recovery $297(48)$; see Figure

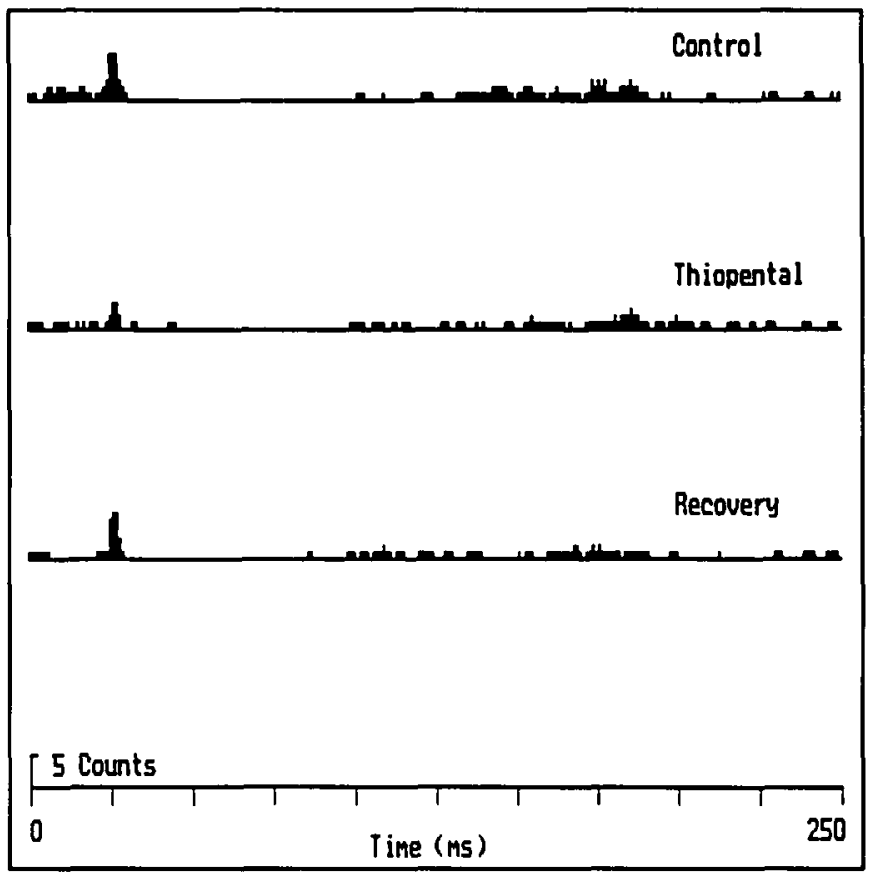

Figure 1: Post stimulus time histograms of the same single motor unit in response to 100 magnetic stimuli over the contralateral motor cortex given 2 ms before time zero. On the control run (top trace) the stimulus over the cortex resulted in a peak of increased firing probability with a latency of $26 \mathrm{~ms}$ and an area of 248 extra coumts. Following intravenous thiopental, total dose $175 \mathrm{mg}$ (middle trace) the peak area is 94 extra counts. After recovery (bottom trace) the peak area is 172 extra coumts. 
2). There was no significant difference between the control and recovery runs so these data were pooled. The mean of the peak areas in the thiopental runs (168 (28)) was significantly less than the mean of the peak areas in the pooled control and recovery runs (317) (48)), $\mathrm{p}<0.025$. There was no significant change in the firing rate of the motor unit between runs (mean control 6.5 $(0.37) \mathrm{Hz}$, thiopental $6.4(0.42) \mathrm{Hz}$, recovery $5.8(0.21) \mathrm{Hz})$. The duration of the peak was not significantly shorter (mean control $3.4(0.68) \mathrm{ms}$, thiopental $2.2(0.25) \mathrm{ms}$, recovery $2.1(0.24) \mathrm{ms})$ and there was no indication that the period of decreased firing probability that follows the peak was proportionally greater after thiopental.

\section{DISCUSSION}

Transcranial stimulation of the cortex provides a new way of examining the action of anesthetic agents on the human brain. There have been a number of such studies (Table) but the information that can be obtained from them depends considerably on the experimental method (Figure 3 ).

Transcranial stimulation of the cortex in man excites corticospinal neurons both directly and indirectly (transsynaptically). The direct activation of corticospinal neurons, which gives rise to direct or " $\mathrm{D}$ " waves in corticospinal axons, probably occurs at the initial segment with magnetic stimulation, while with strong electrical stimulation corticospinal axons appear to be excited deep in the brain. ${ }^{2,10}$ Both forms of stimulation give rise to one or more "I" (indirect) waves in corticospinal axons from transsynaptic activation of corticospinal neurons. These D and I volleys, in turn, generate a composite EPSP in spinal motoneurons which, if sufficiently large, will bring motoneurons to

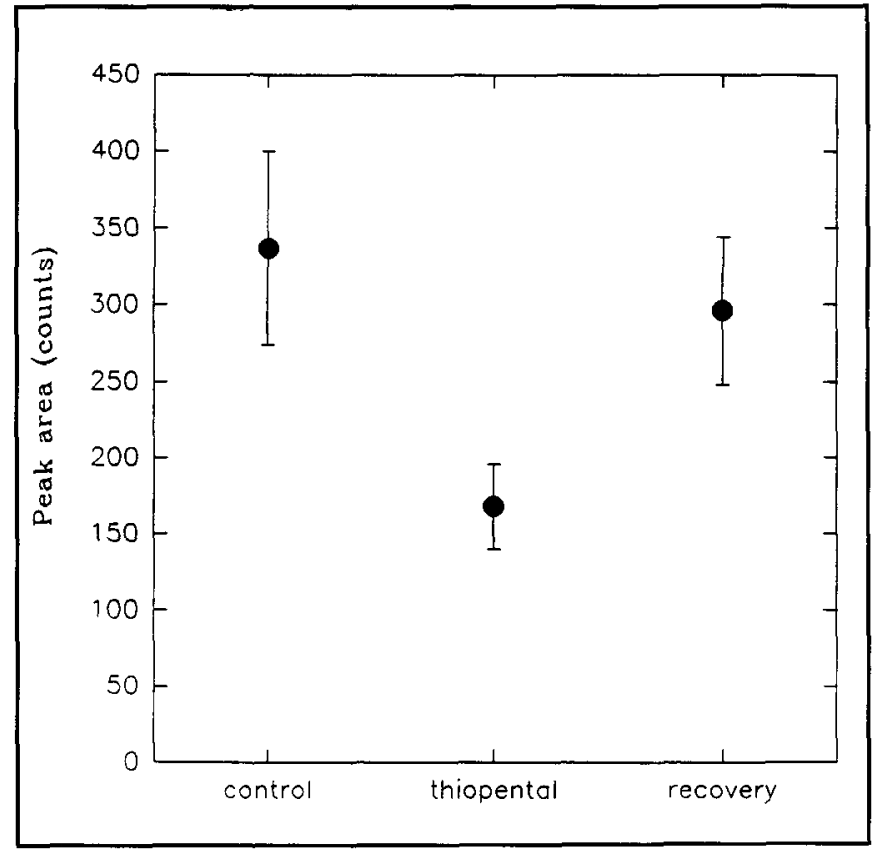

Figure 2: Pooled data from 5 studies showing the mean area ( \pm SE) of the peaks in post stimulus time histograms of single motor units in response to magnetic stimulation over the contralateral motor cortex under control conditions (left), during the infusion of thiopental (middle) and following recovery (right). threshold and result in activation of the muscle which can be recorded as a "motor evoked potential".

The most direct way to use transcranial stimulation to detect changes in the excitability of cortical neurons is to record the $D$ and I waves in corticospinal axons with spinal epidural electrodes. Such studies are limited to circumstances in which it is ethical to place electrodes in the epidural space, for example during spinal surgery. The I waves from magnetic or electrical stimulation and the $\mathrm{D}$ waves from magnetic stimulation would be expected to reflect the excitability of cortical neurons, whereas the D waves to strong electrical stimulation would only reflect changes in the excitability of axons. Such recordings have not been made with thiopental anesthesia but the volatile anesthetic agents isoflurane and enflurane increase the threshold and reduce the amplitude and number of I waves in response to electrical $^{10-12}$ and to magnetic ${ }^{4}$ transcranial stimultaion. Isoflurane can also depress D waves resulting from magnetic stimulation ${ }^{4}$ and from weak electrical stimulation. ${ }^{12}$ The findings imply that

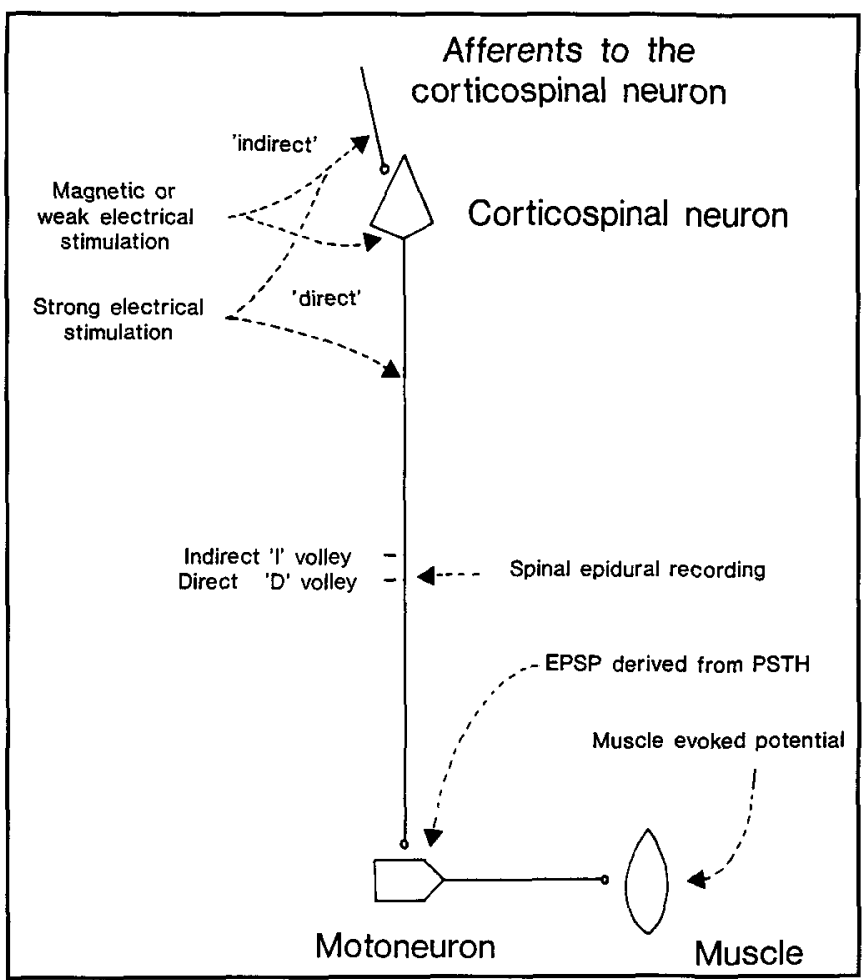

Figure 3: Schematic of the corticospinal system showing the postulated sites of action of transcranial stimulation and sites where evoked activity can be recorded. Magnetic stimulation and weak electrical stimulation excite corticospinal neurons both directly, probably at the initial segment, and indirectly; that is, through axons synapsing onto corticospinal neurons. With strong electrical stimulation the direct activation of corticospinal neurons takes place at their axons deep in the brain. Direct activation of corticospinal neurons gives rise to a " $D$ " volley and indirect activation to one or more " $I$ " volleys in corticospinal axons. $D$ and I waves can be recorded directly with spinal epidural electrodes. The composite excitatory postsynaptic potentials (EPSP) generated by the D and I volleys in single spinal motoneurons can be derived from post stimulus time histograms (PSTH) of single motor units. If the composite EPSP is large enough to bring a number of motoneurons to threshold a muscle compound action potential ("motor evoked potential") can be recorded with surface electrodes over the activated muscle. 
Table: Classification of anesthetic agents and their effects on muscle evoked potentials and on waves recorded from the epidural space following transcranial magnetic or electrical stimulation in humans. The references are numbered in column 7 .

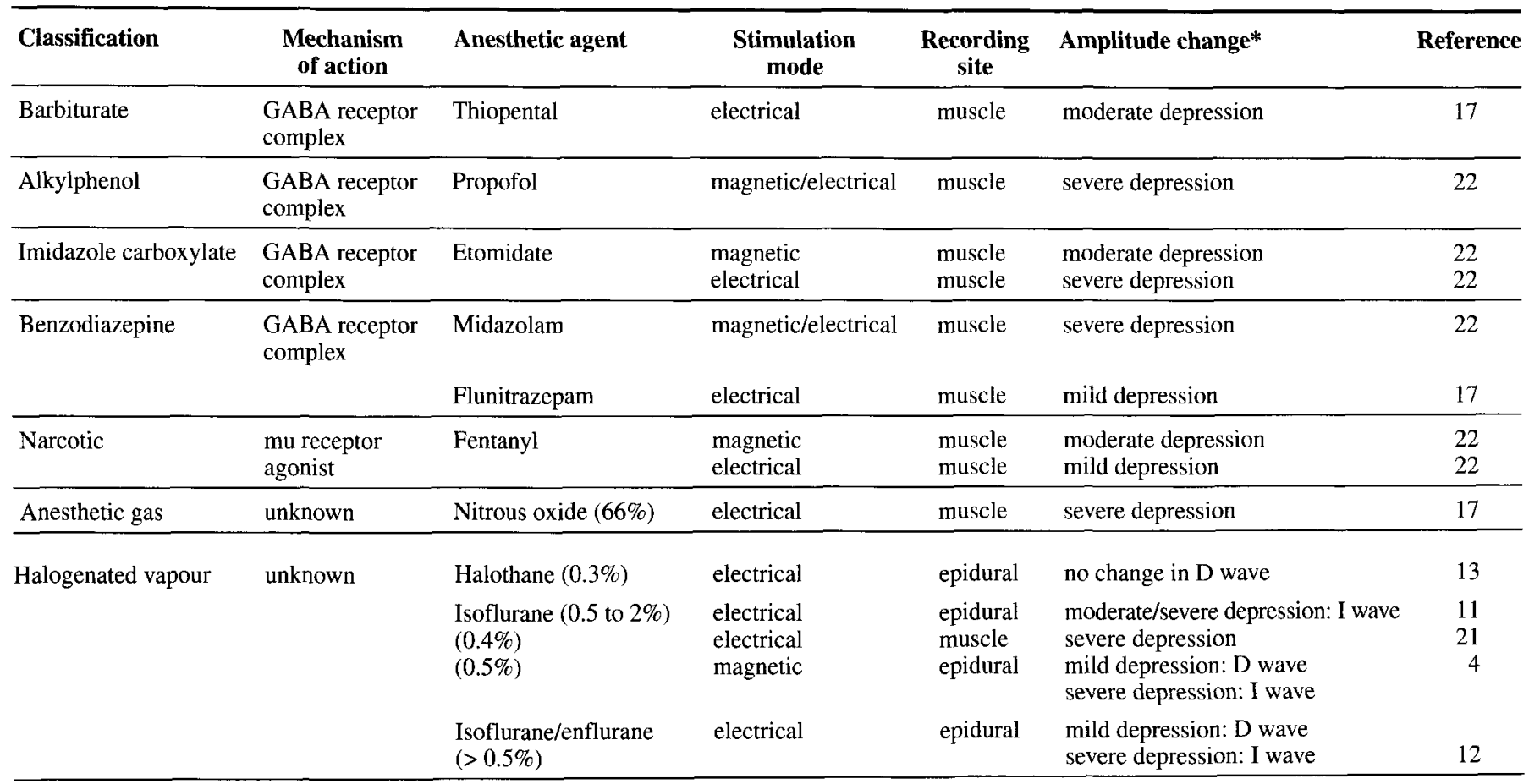

\footnotetext{
*Changes from baseline amplitude:

a) mild depression: $\quad 0-33 \%$ decrease from baseline

b) moderate depression: $34-66 \%$ decrease from baseline

c) severe depression: $\quad 67-100 \%$ decrease from baseline
}

these volatile agents depress the excitability of cortical neurons. On the other hand, the addition of halothane or propofol to nitrous oxide anaesthesia ${ }^{13}$ did not further depress the amplitude of the first (presumably D) wave produced by supramaximal electrical stimulation over the scalp, implying that halothane and propofol do not depress the excitability of corticospinal axons.

In the present study we used a technique which is based on the assumption that the composite EPSP evoked in single spinal motoneurons by a magnetic stimulus over the motor cortex could provide an estimate of the excitability of corticospinalmotoneuron axis. This method is not as invasive as recording spinal evoked potentials but it does require a cooperative subject and not all of our studies were successful. The firing rate of the spinal motoneuron must be kept constant. ${ }^{14}$

We found that composite EPSPs evoked in single spinal motoneurons by magnetic stimulation over the cortex were depressed by thiopental. The most likely explanation for this finding is that thiopental depresses cortical neurons or reduces transmitter release at their terminals. Thiopental is believed to facilitate the action of the inhibitory neurotransmitter GABA probably at multiple sites but certainly including the hippocampal cortex. ${ }^{1}$

Thiopental probably has effects throughout the central nervous system. Could the present findings be explained by an action at the level of the spine? It is possible that the short latency facilitation of spinal motoneurons produced by transcranial stimulation is transmitted, in part, through spinal interneurons which are depressed by thiopental. However, the peaks of increased firing probability produced by magnetic stimulation over the cortex are short enough to be monosynaptic (see discussion in reference 5) and, in the present study, there was no significant reduction in the duration of the peaks of increased firing probability in the runs with thiopental to suggest the loss of an oligosynaptic component. It could be argued that thiopental depresses motoneurons and that the additional corticospinal activity required to maintain their firing rate occluded the volley produced by transcranial stimulation. This seems unlikely as the experiments were carried out with a very weak contraction (a few percent of maximum) implying that, if the cortex is involved in generating a maximum contraction there must be plenty of cortical "reserve". In addition, corticospinal neurons have short refractory periods rendering occlusion unlikely. Finally, it could be argued that, if thiopental increased the conductance of the motoneuron membrane the EPSP in response to a given descending volley would be reduced. Changes in conductance, however, have little effect on large, monosynaptic EPSPs even in motoneurons at rest ${ }^{15}$ and, during rhythmic firing, shunting effects are not seen and only the driving current seems to be important. ${ }^{16}$ Thus this explanation seems unlikely. All of these spinal mechanisms could be excluded by examining the effects of thiopental on the D and I waves recorded with epidural electrodes, by repeating the present experiments with strong anodal electrical stimulation or by recording the changes in firing probability of the same single motoneuron to a segmental input. ${ }^{14}$

A third method of using transcranial stimulation to investigate anesthetic agents is to record the motor potential evoked by 
transcranial stimulation (Table). This potential depends not only on the excitability of cortical neurons but on the excitability of spinal motoneurons, and the function of the endplate and muscle fibers, so such studies cannot distinguish between cerebral, spinal and peripheral sites of actions. Not surprisingly, motor evoked potentials are reduced by thiopental ${ }^{17}$ and it is of interest that Hufnagel et al. ${ }^{18}$ found that the threshold for evoking a motor evoked response was higher in patients who were taking regular oral doses of anticonvulsants (24/53 of whom were taking barbiturates). The motor evoked potential is reduced in rats by isoflurane ${ }^{19}$ and nitrous oxide ${ }^{20}$ and in human subjects by a number of agents (see Table).

We conclude that the present method (particularly with the addition of a segmental input to monitor spinal excitability) may be used to elucidate the actions of drugs on the corticospinalmotoneuronal axis in a non-invasive manner.

\section{ACKNOWLEDGEMENTS}

This study was supported by the Medical Research Council of Canada (Grant \#6727) and the National Institutes of Health (Grant \#27837).

\section{REFERENCES}

1. Olsen RW. Barbiturates. Int Anesthesiol Clin 1988; 26: 254-261.

2. Day BL, Dressler D, Maertens de Noordhout A, et al. Electric and magnetic stimulation of human motor cortex: surface EMG and single motor unit responses. J Physiol 1989; 412: 449-473.

3. Edgley SA, Eyre JA, Lemon RN, Miller S. Excitation of corticospinal tract by electromagnetic and electrical stimulation of the scalp in the macaque monkey. J Physiol 1990; 425: 301-320.

4. Burke D. Hicks R, Gandevia SC, et al. Direct comparison of corticospinal volleys in human subjects to transcranial magnetic and electrical stimulation. J Physiol 1993; 470: 383-393.

5. Palmer E, Ashby P. Corticospinal projections to upper limb motoneurons in humans. J Physiol 1992; 448: 397-412.

6. Ashby P, Zilm D. Relationship between EPSP shape and cross-correlation profile explored by computer simulation for studies on human motoneurons. Exp Brain Res 1982; 47: 33-40.

7. Palmer E, Ashby P. Evidence that a long latency stretch reflex in humans is transcortical. J Physiol 1992; 449: 429-440.
8. Maertens de Noordhout A, Rothwell JC, Day BL, et al. Effect of digital nerve stimulation on responses to electrical or magnetic stimulation of the human brain. J Physiol 1992; 447: 535-548.

9. Mao CC, Ashby P, Wang M, McCrea D. Synaptic connections from large muscle afferents to the motoneurons of various leg muscles in man. Exp Brain Res 1984; 56: 341-350.

10. Burke D, Hicks H, Stephen J. Anodal and cathodal stimulation of the upper-limb area of the human motor cortex. Brain 1992; 115: 1497-1508.

11. Hicks RG, Woodforth IJ, Crawford MR, Stephen JPH, Burke DJ. Some effects of isoflurane on I waves of the motor evoked potential. Br J Anaesth 1992; 69: 130-136.

12. Hicks R, Burke D, Stephen J, Woodforth I, Crawford M. Corticospinal volleys evoked by electrical stimulation of human motor cortex after withdrawal of volatile anaethetics. J Physiol 1992; 456: 393-404.

13. Loughnan BA, Anderson SK, Hetreed MA, et al. Effects of halothane on motor evoked potential recorded in the extradural space. Br J Anaesth 1989; 63: 561-564.

14. Brouwer B, Ashby P, Midroni G. Excitability of corticospinal neurons during tonic muscle contractions in man. Exp Brain Res 1989; 74: 649-652.

15. Edwards FR, Redman SJ, Walmsley B. The effect of polarizing currents on unitary Ia excitatory post-synaptic potentials evoked in spinal neurons. J Physiol 1976; 259: 705-723.

16. Schwindt PC, Calvin WH. Equivalence of synaptic and injected current in determining the membrane potential trajectory during motoneuron rhythmic firing. Brain Res 1973; 59: 389-394.

17. Zentner J, Kiss I, Ebner A. Influence of anaesthetics - nitrous oxide in particular - on electro-myographic response evoked by transcranial electrical stimulation of the cortex. Neurosurgery 1989 24: 253-256.

18. Hufnagel A, Elger CE, Marx W, Ising A. Magnetic motor-evoked potentials in epilepsy: effect of the disease and of anticonvulsant medication. Ann Neurol 1990; 28: 680-686.

19. Haghighi SS, Madsen R, Green KD, Oro JJ, Krackle GR. Suppression of motor evoked potentials by inhalation anesthetics. J Neurosurg Anesthesia 1990; 2: 73-78.

20. Zentner J, Ebner A. Nitrous oxide suppresses the electromyographic response evoked by electrical stimulation of the motor cortex. Neurosurgery $1989 ; 24: 60-62$.

21. Calancie B, Klose KJ, Baier S, Green BA. Isoflurane-induced attenuation of motor evoked potentials caused by electrical motor cortex stimulation during surgery. J Neurosurg 1991; 74: 897-904.

22. Kalkman CJ, Drummond JC, Ribberink AA, et al. Effects of propofol, etomidate, midazolam and fentanyl on motor evoked responses to transcranial electrical or magnetic stimulation in humans. Anesthesiology 1992; 76: 502-509. 